\section{Reactive arthritis in a patient with cat-scratch disease}

Sir,

Arthropathy has rarely been reported as a feature of cat-scratch disease. We report a patient who developed a poly irthritis as a manifestation of this condition. A 39 year old male designer presented with a 4 week history of painful swelling in the right groin and a maculo-papular rash on the right thigh which has failed to improve with oral flucloxacillin therapy. He was otherwise well with no significant medical history though his 7 year old son had recently had an excision biopsy of a neck gland. He had a kitten and admitted to having been scratched as had his son. Examination revealed a papular rash over the right anterior thigh and lymphadenitis in the right groin with an overlying pustule but no other lymphadenopathy. Pus was aspirated and excision biopsy of the node was performed, histology revealing active changes and multiple necrotizing granulomata. No acid-fast bacilli were identified and culture was negative. Although Dieterle and Warthin-Starry staining did not reveal any organisms, the histology was felt to be strongly indicative of cat-scratch disease and the patient fulfilled the necessary three of four criteria of: (1) history of animal contact (especially cats or kittens) with presence of a scratch or primary dermal or ocular lesion; (2) laboratory data negative for other infectious causes of lymphadenopathy; (3) node biopsy revealing histopathology consistent with cat-scratch disease and/or demonstration of typical organisms using Warthin-Starry silver preparation; and (4) positive cat-scratch skin test (available only in the USA at the time).' His son's excised lymph node showed similar histology with multiple necrotizing granulomata.

Three days after excision of the lymph node the patient developed severe pain in the left wrist, right thumb and right ankle, and generalized aching and stiffness in ankles, knees, wrists and hands. Symptoms were helped to a minor degree by soluble aspirin.
Examination revealed acute synovitis of the right wrist and right ankle with tenderness over bilateral metacarpophalangeal and left ankle joints. The patient was pyrexial with a temperature of $38^{\circ} \mathrm{C}$.

$\mathrm{X}$-ray of chest and sacro-iliac joints, full blood count and differential white count, biochemistry, rheumatoid factor and autoantibody screen, complement levels and electrophoresis of plasma proteins were all normal. Erythrocyte sedimentation rate was $26 \mathrm{~mm} /$ hour, plasma viscosity 1.77 (normal range $1.5-1.72$ ). He did not have the HLA B27 phenotype. Toxoplasma serology gave a titre of 160 and chlamydial serology a titre of 512 but there was no change in paired samples suggesting previous exposure but no recent infection.

The patient was treated with non-steroidal antiinflammatory drugs and improved dramatically and in the 2 years since then has had only infrequent aches and pains in wrists and elbows with no further episodes of synovitis.

Polyarthritis is a rare manifestation of cat-scratch disease and we are aware of only one previous report which was limited to bilateral ankle arthritis, ${ }^{2}$ though arthralgia of the shoulder, elbow and wrist in a child with cat scratch disease has been reported. ${ }^{3}$

$$
\begin{array}{r}
\text { M.M. Arnold } \\
\text { F. McKenna } \\
\text { Rheumatic Diseases Unit, } \\
\text { Trafford General Hospital, } \\
\text { Davyhulme, } \\
\text { Manchester M31 3SL, UK. }
\end{array}
$$

\section{References}

1. Carrithers, H.A. Cat-scratch disease: overview based on a study of 1,200 patients. Am J Dis Child 1985, 139: 1124-1233.

2. Goddard, N.J. \& Golding, D.N. Cat-scratch disease presenting with arthropathy of the ankles. $J R$ Soc Med 1989, 82: 499-500.

3. Fowler, R.S. \& Bailey, J.D. Cat-scratch disease in childhood. Can Med Assoc J 1961, 84: 1365-1368. 